\title{
Avaliação da qualidade dos serviços de atenção primária à saúde acessados por idosos em dois distritos de Porto Alegre, RS, Brasil
}

\section{Quality assessment of primary health care accessible to the elderly in two districts of Porto Alegre, RS, Brazil}

Júlia de Melo Dotto* Gabriela Adima de Camargo Ávila*

Aline Blaya Martins ${ }^{* *}$

Fernando Neves Hugo**

Otávio Pereira D'Avila**

Juliana Balbinot Hilgert ${ }^{* * * *}$

\section{Resumo}

Objetivo: avaliar a orientação dos serviços de Atenção Primária à Saúde (APS) e comparar a qualidade da APS entre Unidades Básicas de Saúde (UBSS) e Unidades de Saúde da Família (USFs) em dois distritos de Porto Alegre, Rio Grande do Sul. Sujeitos e método: neste estudo de delineamento transversal, foram entrevistados 402 idosos, selecionados por meio de uma amostra por conglomerados. Foi aplicado um questionário com questões sociodemográficas, além do instrumento Primary Care Assessment Tool (PCATool-Brasil). Atributos com escores maiores ou iguais a 6,6, em uma escala de 0 a 10, foram considerados orientados para a APS. Foi realizada análise descritiva das variáveis, e a diferença entre as variáveis entre UBSs e USFs foi verificada pelo teste Qui-Quadrado. Resultados: dos 402 entrevistados, $69,7 \%$ eram do sexo feminino, as médias encontradas foram: de idade, 69,18 anos; de anos de estudo, 4,91; de renda, $1.433,84$ reais. A maioria $(77,9 \%)$ utilizava os serviços de UBS e $22,1 \%$ utilizavam USF.

Quanto à qualidade dos serviços, 22,9\% estavam dentro do preconizado pelo escore essencial da APS. Quando comparadas as UBSs e as USFs, houve diferença estatisticamente significativa em relação à idade ( $\leq 70$ anos acessavam $71,9 \%$ USFs X 58,3\% UBSs) e aos atributos: primeiro contato (39,7\% UBSs X 51,7\% USFs com orientação para APS, $p=0,044)$ e longitudinalidade $(68,5 \%$ USFs X 50\% UBSs com orientação para APS, $p=0,02$ ). Conclusão: os resultados evidenciam a necessidade de investimentos para a qualificação da APS, pois, embora a Estratégia de Saúde da Família seja prioridade na reorganização da APS, essa demonstrou superioridade em apenas dois atributos: primeiro contato e longitudinalidade.

Palavras-chave: Atenção Primária à Saúde. Epidemiologia dos serviços de saúde. Idoso. Serviços de saúde para idosos.

Cirurgiã-dentista. Faculdade de Odontologia da Universidade Federal do Rio Grande do Sul, Porto Alegre, Brasil.

Doutor(a) em Saúde Bucal Coletiva. Docente da Faculdade de Odontologia da Universidade Federal do Rio Grande do Sul, Porto Alegre, Brasil.

Mestre em Saúde Bucal Coletiva. Doutorando do Programa de Pós-Graduação da Faculdade de Odontologia da Universidade Federal do Rio Grande do Sul, Porto Alegre, Brasil.

***** Doutora em Epidemiologia. Docente da Faculdade de Odontologia da Universidade Federal do Rio Grande do Sul, Porto Alegre, Brasil. 


\section{Introdução}

No Brasil, a expectativa de vida vem aumentando de modo significativo. Concomitantemente, há uma redução das taxas de fertilidade, de modo que a pirâmide etária populacional vem mudando de sua forma triangular, de 2002, para, possivelmente, uma forma com ápice mais largo, prevista para 2025. Dessa forma, é preciso criar medidas de atenção à saúde que possibilitem às pessoas envelhecer com saúde e ter uma vida mais ativa ${ }^{1}$.

Para o ano de 2050, estima-se que mais de metade das pessoas com 60 anos ou mais viverão em países em desenvolvimento. nesse contexto, o Brasil terá a sexta maior população de idosos do mundo, possivelmente, passando de 30 milhões já em $2025^{2}$.

A Atenção Primária à Saúde (APS) define-se por um conjunto de valores, princípios e elementos estruturantes do sistema de serviços de saú$\mathrm{de}^{3}$, que busca atender a maioria dos problemas da população, incluindo o grupo dos idosos, devendo, portanto, estar adequado às necessidades das pessoas idosas, que apresentam, em sua maioria, doenças crônicas. Em Porto Alegre, coexistem dois modelos de saúde: um direcionado à fragmentação das diferentes áreas, com foco na cura da doença, denominado Unidades Básicas de Saúde (UBSs) tradicionais, e o outro direcionado ao cuidado com foco na saúde da família, Unidades de Saúde da Família (USFs).

As UBSs abrangem um território maior, em que não há adscrição da clientela e têm uma prática bastante limitada no que tange à vigilância à saúde, visto que não há agentes comunitários de saúde. Enquanto que as USFs fundamentam-se na Estratégia de Saúde da Família (ESF), tendo equipes multiprofissionais, trabalho multi e interdisciplinar, adscrição da população, mantendo o foco da atenção à saúde da família.

Como há necessidade de avaliar a qualidade dos serviços de APS, foi criado o Instrumento de Avaliação da Atenção Primária à Saúde, o Primary Care Assessment Tool (PCATool) ${ }^{4}$. O PCATool original ${ }^{5,6}$ foi adaptado e validado para o uso no Brasil em 2010, passando então a ser denominado PCATool-Brasil.

Em suma, tendo em vista o fenômeno de envelhecimento da população e as políticas que preconizam o cuidado prioritário dessa faixa etária, visando contribuir para que o Sistema Único de Saúde dê respostas efetivas e eficazes às necessidades e às demandas de saúde da população brasileira, este estudo avaliou, por meio do instrumento PCATool-Brasil, a orientação dos serviços de Atenção Primária à Saúde acessados por idosos residentes em dois distritos de Porto Alegre, de acordo com os atributos da APS, e, também, comparou as diferenças de adequação entre UBSs e USFs.

\section{Sujeitos e método}

Este estudo trata-se de uma pesquisa quantitativa descritiva de base populacional, no qual o delineamento da pesquisa é do tipo observacional transversal, em que foram analisados dados de 402 participantes.

Foram convidados a participar do estudo idosos que vivem na comunidade de forma independente, com 60 anos de idade ou mais, moradores dos distritos sanitários Lomba do Pinheiro e Partenon, em Porto Alegre, Rio Grande do Sul.

Esses dois distritos sanitários foram escolhidos para a aplicação de um questionário de pesquisa (PCATool-Brasil) a fim de verificar a extensão em que o cuidado é produzido conforme as condições referidas pelos idosos pesquisados. Tais distritos são vinculados à rede pública municipal. Segundo o Censo IBGE 2010, Porto Alegre tinha uma população de 1.409.939 habitantes, sendo 211.986 pessoas com idade de 60 anos ou mais. Nos referidos distritos, a população estimada com essa faixa etária era de 12.871 indivíduos ${ }^{7}$.

Os critérios para inclusão dos sujeitos da pesquisa foram: ter 60 anos ou mais, ser morador dos distritos sanitários Lomba do Pinheiro e Partenon, em Porto Alegre, e não apresentar déficit cognitivo segundo o teste de rastreamento Mini Exame do Estado Mental (MEEM) ${ }^{8}$. A fim de rastrear possíveis danos cognitivos que comprometessem a qualidade dos dados, diferentes pontos de corte foram utilizados como critério de inclusão. A saber, ao realizar-se a soma das respostas dos trinta itens que compõem o MEEM (contando 1 ponto por acerto), o número mínimo de acertos para inclusão na amostra foi de: 13 para analfabetos, 18 para escolaridade média ( 1 a 8 anos de estudo) e 26 para alta escolaridade (9 anos de estudo ou mais $)^{8}$.

Dessa forma, somente aqueles idosos que tinham 60 anos ou mais, viviam de forma independente (conseguiam realizar as atividades rotineiras sem o auxílio de cuidadores), aceitaram participar do estudo, assinaram o Termo de Consentimento Livre e Esclarecido e atingiram escores do MEEM acima do ponto de corte foram convidados a responder os demais instrumentos que fizeram parte das medidas avaliadas neste estudo.

Para estimação do tamanho amostral, foi realizado um estudo piloto com cinquenta idosos moradores dos distritos que foram avaliados nesta pesquisa. O cálculo amostral foi realizado considerando a prevalência de autopercepção de saúde bucal boa, muito boa ou excelente, que foi igual a $59 \%$. Estimou-se que a razão de prevalência dos idosos que dispunham de uma atenção primária adequada foi de 1,32, conforme o escore essencial do Instrumento de Avaliação da Atenção Primária (PCATool-Brasil) ${ }^{9}$. 
Assim, selecionado um alfa bidirecional de 5\% e um beta de $20 \%$, foi estimada uma amostra total de 454 idosos. Foram acrescentados $10 \%$ para possíveis perdas ou recusas e $15 \%$ para controle de fatores de confusão. Na falta de uma estimativa precisa para minimizar o efeito do delineamento, que ocorre no processo amostral por conglomerados, a amostra foi ampliada 1,5 vez para realizar as análises ajustadas. Chegou-se, assim, ao número de 883 indivíduos.

Assim, a amostragem foi realizada por meio da metodologia proposta por Barros e Victora ${ }^{10}$ para avaliação de conglomerados (setores censitários), sendo que o número de setores censitários é proporcional ao total de setores censitários nos dois distritos nos quais o estudo foi realizado. $\mathrm{O}$ número de domicílios visitados foi estimado a partir do número médio de moradores em cada domicílio e da proporção de idosos na cidade de Porto Alegre em 2010. Tais informações foram obtidas na Coordenação Geral de Vigilância em Saúde do Município de Porto Alegre. Dentro de cada distrito, foram amostrados os setores censitários, utilizando-se uma amostragem sistemática, a fim de garantir a heterogeneidade que se expressa na ocupação do espaço.

O projeto foi aprovado pelos Comitês de Pesquisa e de Ética da Faculdade de Odontologia da Universidade Federal do Rio Grande do Sul no dia 18 de junho de 2009 , ata $\mathrm{n}^{\circ}$ 05/09, com o número do processo 15.297.

Previamente à coleta de dados, foi realizado um treinamento com a equipe de entrevistadores, graduandos e pós-graduandos. Inicialmente, foram convidados potenciais participantes, aos quais foram esclarecidos os objetivos do estudo. Aqueles que aceitaram participar e assinaram o termo de consentimento informado responderam a um questionário de identificação e ao MEEM. A partir disso, apenas participantes que cumpriram os critérios de inclusão foram convidados a responder o questionário padronizado composto pelo conjunto de medidas que foram avaliadas neste estudo e realizaram um breve exame de saúde bucal.

Com a aplicação de um questionário individual, foram coletadas informações a respeito de sexo, idade, escolaridade, renda, número de dependentes dessa e percepção de que a renda era suficiente para a família. A idade, a escolaridade (número de anos completados de educação formal) e o número de dependentes da renda familiar foram coletados como variáveis contínuas e expressas em forma de média e desvio padrão.

A variável hábito tabágico foi investigada pela pergunta: “O(a) sr.(a) fuma atualmente?", para a qual o sujeito poderia responder positiva ou negativamente. Já a variável hábito etílico foi avaliada por meio da seguinte questão: "Com que frequência o(a) sr.(a) consome bebidas alcoólicas?”, com respostas variáveis entre nunca e quatro ou mais vezes por semana. Tais respostas foram categorizadas, para fins de análise, da seguinte maneira: nunca, raramente (no máximo 1 vez no mês), eventualmente ( 2 a 4 vezes por mês), frequentemente ( 2 a 3 vezes por semana, 4 vezes ou mais por semana).

Trata-se de um instrumento de rastreamento de possíveis déficits cognitivos, desenvolvido por Folstein et al. ${ }^{11} \mathrm{e}$ validado para o português brasileiro ${ }^{8}$.

Dentre os indivíduos que foram incluídos na amostra para a análise da função cognitiva, maiores escores foram considerados como melhor desempenho cognitivo. Para tanto, as médias dos escores do MEEM de cada nível de escolaridade foram obtidas e uma variável foi criada, estabelecendo a média, de acordo com escolaridade baixa, média ou alta, como ponto de corte. Foram, então, definidas as seguintes categorias: acima da média do MEEM - capacidade cognitiva alta e abaixo da média do MEEM - capacidade cognitiva baixa.

Informações acerca de diabetes, hipertensão e dor nas costas foram coletadas com base na seguinte pergunta: "Das doenças que vou lhe perguntar a seguir, responda sim caso algum médico já tenha lhe dito que o(a) senhor(a) tem".

O Instrumento de Avaliação da Atenção Primária foi criado ${ }^{5,6}$ e validado para o idioma português brasileiro e proposto pelo Ministério de Saúde ${ }^{9}$ como instrumento de avaliação da qualidade de serviços de saúde de atenção primária no Brasil. O instrumento tem três versões voltadas a avaliar a APS a partir do ponto de vista de diferentes atores: usuários, profissionais e infantil (pais ou responsáveis).

O instrumento PCATool para usuários é composto por 55 itens divididos em dez componentes (subescalas) relacionados da seguinte forma: grau de afiliação com serviço de saúde; acesso de primeiro contato - utilização; acesso de primeiro contato - acessibilidade; longitudinalidade; coordenação integração de cuidados; coordenação - sistema de informações; integralidade - serviços disponíveis; integralidade - serviços prestados; orientação familiar; orientação comunitária.

As respostas são do tipo Likert com 4 pontos, variando de 1 (com certeza não) a 4 (com certeza sim). $\mathrm{O}$ instrumento atribui, por meio das subescalas, escores para cada atributo essencial, a saber: acesso de primeiro contato, longitudinalidade, integralidade e coordenação do cuidado. E para os atributos derivados: orientação familiar e orientação comunitária. O cálculo dos escores seguiu as orientações do manual de uso do instrumento ${ }^{2}$. Dessa forma, atributos com escores maiores ou iguais a 6,6 , em uma escala de 0 a 10 , foram considerados orientados para a APS.

Questionário com informações sobre os serviços acessados, incluindo o tipo de serviço utilizado em relação à saúde geral e o nível de satisfação com esse serviço. Dentre os tipos de serviços de saúde que costumavam ser acessados estavam: Unidade 
Básica de Saúde - que costumam operar segundo o modelo tradicional biomédico; Unidade de Saúde da Família - que seguem o modelo baseado nos atributos da APS; ambulatórios de convênio; médicos ou serviços particulares; hospitais, entre outros atendimentos. Para fins de análise estatística, os tipos de serviços utilizados neste estudo foram somente unidades de saúde de APS: UBS e USF.

Ao final da coleta, os dados foram tabulados e analisados no software SPSS 18.0, para análises estatísticas (SPSS Inc., USA). As variáveis contínuas e discretas foram descritas na forma de média e desvio padrão, enquanto as variáveis categóricas foram descritas na forma de porcentagens absoluta e relativa.

A confiabilidade das escalas Mini Exame do Estado Mental e das subescalas que compõem o PCATool-Brasil foi testada por meio do Coeficiente Alpha de Cronbach.

\section{Resultados}

Foram convidados a participar deste estudo, durante o período de agosto de 2010 a fevereiro de 2011, 890 idosos residentes na gerência Lomba-Partenon. Dos convidados, 53 não foram encontrados nos domicílios no dia do exame e não conseguiram ser agendados após três tentativas, 10 se recusaram a participar e 23 não atingiram os pontos de corte do MEEM. A amostra do estudo, portanto, foi composta por 862 pessoas que assinaram o Termo de Consentimento Livre e Esclarecido, aceitaram participar do estudo e estavam aptos a compor a amostra. Do total de participantes do estudo, apenas 401 idosos utilizavam os serviços de APS, razão pela qual foram incluídos nesta análise.

A amostra estudada foi composta por 122 homens $(30 \%)$ e 280 mulheres $(69,7 \%)$, a média de idade dos participantes foi de 69 anos $( \pm 6,84)$ e a escolaridade média foi de aproximadamente 5 anos de estudos completos $( \pm 3,39)$. A renda familiar média foi de $R \$ 1.433,84( \pm R \$ 1.048,00)$, tendo sido a mediana igual a $R \$ 1.100,00$, sendo que, em metade das famílias, no mínimo três pessoas, incluindo o idoso participante da pesquisa, dependiam dessa renda para sua subsistência, e $49,4 \%$ dos idosos referiram que achavam que a renda familiar não era suficiente para suprir as necessidades das suas famílias (Tabela 1).
Tabela 1 - Frequências das variáveis sociodemográficas

\begin{tabular}{l|l|r}
\hline \multicolumn{1}{c|}{ Variáveis } & & $\mathrm{n}(\%) / \mathrm{m}(\mathrm{dp})$ \\
\hline Idade & & $69,18(5,85)$ \\
Renda familiar & & $1433,84(1048,00)$ \\
Anos de estudo & & $4,91(3,39)$ \\
Sexo & Masculino & $122(30,3)$ \\
& Feminino & $280(69,7)$ \\
Renda suficiente & Sim & $123(30,6)$ \\
& Não & $279(69,4)$ \\
Situação conjugal & Casado & $179(44,9)$ \\
& Não casado & $220(55,1)$ \\
Participação em & Sim & $48(12)$ \\
grupo de idosos & Não & $353(88)$ \\
\hline Fonte: dados da pesquisa &
\end{tabular}

Fonte: dados da pesquisa.

As condições de saúde geral dos idosos que fizeram parte dessa amostra foram: 39,3\% apresentaram capacidade cognitiva abaixo da média, considerando o escore do MEEM e os anos de escolaridade; $60 \%$ referiram ser hipertensos; $22 \%$ referiram ser diabéticos; $44,6 \%$ referiram sentir dor nas costas; $34,5 \%$ apresentaram sintomas depressivos segundo a escala GDS; $74,3 \%$ perceberam a própria saúde como ruim.

Em relação ao uso dos serviços, $22,2 \%$ dos idosos acessam, habitualmente, Unidades de Saúde da Família e 77,8\% são usuários de Unidades Básicas de Saúde. No que tange à satisfação com os serviços acessados, $22 \%$ dos idosos não recomendariam o serviço de saúde que costumam acessar para uma pessoa de sua família.

Quanto à qualidade de tais serviços, o valor médio de escore dos atributos foi: i) primeiro contato: 6,27 ; ii) coordenação: 5,20 ; iii) integralidade: 4,46 ; iv) longitudinalidade: 6,$42 ;$ v) escore essencial: 5,64. Quanto à qualidade dos serviços, $23 \%$ estavam dentro do preconizado pelo escore essencial da APS. Analisando a orientação de cada atributo individualmente, tiveram orientação para a APS: $54 \%$ dos serviços em relação à longitudinalidade; $42,3 \%$ em relação ao primeiro contato; 44,5\% em relação à coordenação do cuidado; $24 \%$ em relação à integralidade (Tabela 2 ).

Tabela 2 - Frequências das variáveis relacionadas com a orientação para APS dos serviços acessados

\begin{tabular}{l|l|r}
\hline Variáveis & & $\mathrm{n}(\%) / \mathrm{m}(\mathrm{dp})$ \\
\hline Uso APS & USF & $89(22,2)$ \\
& UBS & $312(77,8)$ \\
Escore 1ํo contato & & $6,27(1,25)$ \\
Escore coordenação & & $5,20(1,92)$ \\
Escore integralidade & & $4,46(1,63)$ \\
Escore longitudinalidade & & $6,42(1,43)$ \\
Escore essencial & & $5,64(1,13)$ \\
\hline Fonte: dados da pesquisa &
\end{tabular}

Fonte: dados da pesquisa. 
Quando comparados, os serviços de UBSs e USFs, em relação aos dados sociodemográficos, não tiveram diferença significativa, com exceção da variável idade ( $\leq 70$ anos, $71,9 \%$ USFs X 58,5\% UBSs, $\mathrm{p}=0,020$ ). Foram associados estatisticamente, de acordo com os modelos de atenção, os escores dos seguintes atributos: primeiro contato (com orientação para APS, $51,7 \%$ USFs X 39,7\% UBSs, $\mathrm{p}=0,044)$ e longitudinalidade (com orientação para APS, $68,5 \%$ USFs X 50\% UBSs, $p=0,002$ ) (Tabela 3).

Tabela 3 - Distribuição das frequências das variáveis preditoras em relação ao uso de diferentes modelos de APS

\begin{tabular}{|c|c|c|c|c|}
\hline \multicolumn{2}{|c|}{ Variáveis } & \multirow{2}{*}{$\frac{\text { USF } n(\%)}{64(71,9)}$} & \multirow{2}{*}{$\frac{\text { UBS } n(\%)}{183(58,3)}$} & \multirow[t]{2}{*}{ P-valor } \\
\hline Idade & $\leq 70$ anos & & & \\
\hline & $\geq 71$ anos & $25(28,1)$ & $130(41,7)$ & 0,020 \\
\hline \multirow[t]{2}{*}{ Renda familiar } & $\leq 2$ salários $(\$ 1090,00)$ & $49(55,1)$ & $155(49,5)$ & \\
\hline & >2 salários $(\$ 1091,00)$ & $40(44,9)$ & $158(50,5)$ & 0,357 \\
\hline \multirow[t]{2}{*}{ Anos de estudo } & $\leq 6$ anos & $66(74,2)$ & $236(75,6)$ & \\
\hline & $\geq 7$ anos & $23(25,8)$ & $76(24,4)$ & 0,775 \\
\hline \multirow[t]{2}{*}{ Sexo } & Masculino & $25(28,1)$ & $97(31,1)$ & \\
\hline & Feminino & $64(71,9)$ & $215(68,9)$ & 0,587 \\
\hline \multirow[t]{2}{*}{ Renda suficiente } & $\operatorname{Sim}$ & $31(34,8)$ & $92(29,5)$ & \\
\hline & Não & $58(65,2)$ & $220(70,5)$ & 0,335 \\
\hline \multirow[t]{2}{*}{ Situação conjugal } & Casado & $45(50,6)$ & $134(43,2)$ & \\
\hline & Não Casado & $44(49,4)$ & $176(56,8)$ & 0,220 \\
\hline \multirow[t]{2}{*}{ Participação em grupo de idosos } & $\operatorname{Sim}$ & $10(11,2)$ & $38(12,2)$ & \\
\hline & Não & $79(88,8)$ & $274(87,8)$ & 0,809 \\
\hline \multirow[t]{2}{*}{ Religião } & Católico & $46(51,7)$ & $180(57,7)$ & \\
\hline & Outras Crenças & $43(48,3)$ & $132(42,3)$ & 0,313 \\
\hline \multirow[t]{2}{*}{ Escore $1^{\circ}$ contato } & Sem orientação APS & $43(48,3)$ & $188(60,3)$ & \\
\hline & Com orientação APS & $46(51,7)$ & $124(39,7)$ & 0,044 \\
\hline \multirow[t]{2}{*}{ Escore coordenação } & Sem orientação APS & $43(49,4)$ & $176(56,6)$ & \\
\hline & Com orientação APS & $44(50,6)$ & $135(43,4)$ & 0,235 \\
\hline \multirow[t]{2}{*}{ Escore integralidade } & Sem orientação APS & $68(76,4)$ & $237(76)$ & \\
\hline & Com orientação APS & $21(23,6)$ & $75(24)$ & 0,931 \\
\hline \multirow[t]{2}{*}{ Escore longitudinalidade } & Sem orientação APS & $28(31,5)$ & $156(50)$ & \\
\hline & Com orientação APS & $61(68,5)$ & $156(50)$ & 0,002 \\
\hline \multirow[t]{2}{*}{ Escore essencial } & Sem orientação APS & $62(69,7)$ & $247(79,2)$ & \\
\hline & Com orientação APS & $27(30,3)$ & $65(20,8)$ & 0,060 \\
\hline
\end{tabular}

Fonte: dados da pesquisa.

\section{Discussão}

Há poucos estudos comparativos em relação à qualidade da APS que analisem os modelos assistenciais de acordo com a percepção dos usuários ${ }^{12}$. Em Porto Alegre, foi encontrado apenas um estudo feito com adultos ${ }^{13}$, dessa forma, este estudo é pioneiro em relação à abordagem com idosos. Sendo assim, torna-se difícil mensurar o grau de êxito dos diferentes tipos de serviços de saúde bem como definir qual está mais próximo do ideal de acordo com os princípios da APS e, principalmente, com as necessidades das pessoas idosas. Não obstante, a ESF tem princípios orientados à APS, por isso espera-se que esse tipo de serviço esteja mais próximo do preconizado pela Política Nacional de Atenção Básica. $\mathrm{O}$ resultado encontrado neste estudo demonstrou maior orientação da ESF para APS no que tange aos atributos longitudinalidade e primeiro contato. De fato, foi observado que as USFs apresentaram maior orientação à APS quando comparadas com as UBSs tradicionais.

Em relação à variável sociodemográfica idade, os resultados deste estudo mostraram diferença significativa no acesso a diferentes modelos de atenção em saúde, quando foram comparadas pessoas com 70 anos ou menos de idade ou 71 anos ou mais. Sendo que aqueles que tinham 70 anos ou menos procuram mais a USF do que a UBS. Pode-se especu- 
lar que o histórico de barreiras de acesso ao serviço somado a um acúmulo de doenças, principalmente crônicas, desenvolveram o hábito de os idosos mais velhos e expostos por mais tempo a essa realidade desenvolverem uma rotina de busca de serviços que atuem por livre demanda e sem territorialização. Ambas as características são comuns às USFs, que promovem mudanças na organização do processo de trabalho das equipes e no acesso aos serviços nem sempre bem aceito pelos mais velhos.

Os atributos da APS são reconhecidos internacionalmente como eixo estruturante do processo de trabalho na atenção básica ${ }^{5,9} \mathrm{e}$, para isso, seguem algumas características organizacionais, tais como: acesso facilitado dos usuários, continuidade e integralidade do cuidado, responsabilização das equipes frente às comunidades atendidas, formação de vínculo entre profissionais e usuários e fluxo dos usuários entre os diferentes níveis de atenção ${ }^{14}$. Neste estudo, nenhum dos valores médios dos escores atributos atingiu o mínimo necessário $(6,6)$ para considerar que estavam orientados para APS, sendo que o atributo primeiro contato foi o que chegou mais próximo, atingindo a média de 6,27. Embora não se tenha encontrado estudos que aplicaram PCATool em idosos. Esse resultado está de acordo com o que foi observado por Oliveira ${ }^{13}$, ao aplicar o PCATool em uma população de adultos, maiores de 18 anos de idade, de Porto Alegre, onde os valores médios dos atributos essenciais também ficaram abaixo de 6,6 ao se comparar UBS e ESF, com exceção do atributo primeiro contato, que estava orientado para APS tanto em UBS $(8,2)$ quanto em $\operatorname{ESF}(8,9)$.

Em relação ao atributo primeiro contato, foram encontrados resultados que evidenciam que ainda há muito a melhorar em relação ao acesso universal das pessoas idosas à APS, pelo menos no que se refere aos idosos que participaram deste estudo, uma vez que menos da metade dos serviços atingiu o escore mínimo de orientação para a APS. Quando comparados os distintos modelos de APS, encontra-se resultados mais favoráveis no modelo voltado para a saúde da família. O atributo primeiro contato implica acessibilidade e utilização dos serviços pelos usuários para cada novo problema de saúde ou para cada novo episódio de problema de saúde. $\mathrm{O}$ serviço só oferece atenção ao primeiro contato quando os seus usuários o perceberem como acessível ${ }^{4}$.

Sendo assim, ao encontrar $51,7 \%$ dos estabelecimentos de saúde da família com orientação à APS, contra $39,7 \%$ das UBSs, percebe-se que ainda será necessário ampliar bastante o acesso aos serviços dessas unidades, a fim de que a atenção básica realmente possa ser apontada como a porta preferencial de entrada ao serviço de saúde.

Observando essa diferença, entretanto, ao comparar os modelos, observa-se uma diferença significativa em relação a esse atributo, em que as USFs mostraram melhor orientação que as UBSs. Credita-se tal diferença ao fato de que as equipes de saú- de da família apresentam uma lógica de atenção à saúde voltada a um número limitado de pessoas e processos de trabalho, na qual agentes comunitários e demais profissionais de saúde procuraram estabelecer uma constante busca ativa e não atender apenas a demanda espontânea. Ou seja, a equipe empenha-se em ampliar a atenção à saúde por meio de dispositivos, como as visitas e os atendimentos domiciliares realizados pela equipe, e, assim, cria-se uma forma de proporcionar a utilização do serviço por parte de usuários idosos que se encontram acamados ou com outras limitações físicas ou cognitivas. De acordo com Gomes et al. ${ }^{15}$, os agentes comunitários de saúde, por meio da busca ativa e de visitas domiciliares, são importantes para proporcionar maior acesso dos usuários às USFs, o que vai ao encontro do que é o defendido por este estudo.

A longitudinalidade, de acordo com o conceito na APS, refere-se a uma relação pessoal de longa duração entre profissionais e usuários dos serviços de saúde, podendo haver interrupções no processo, mas não nessa relação ${ }^{3}$. Esse atributo reflete o quanto o serviço está abordando uma atenção continuada, demostrando essa identificação mútua entre profissionais de saúde e usuários. Os resultados desse estudo, no que se refere a esse atributo, também demostraram maior orientação para APS nas USFs quando comparadas com as UBSs.

É possível que tal resultado seja consequência de diferentes abordagens em relação à continuidade do cuidado, visto que a ESF se propõe a trabalhar com prevenção e educação para a saúde perpassando todo o ciclo de vida dos indivíduos ${ }^{1}$, obtendo, assim, maior probabilidade de amenizar futuros problemas de saúde e também maior conhecimento, proximidade e envolvimento com as famílias ${ }^{16}$. $\mathrm{O}$ vínculo longitudinal auxilia na obtenção de diagnósticos e tratamentos mais precisos, maior satisfação do indivíduo e diminuição de internações por condições crônicas, visto que o tratamento pode ser feito no nível ambulatorial ${ }^{17}$. Também proporciona melhor aceitação de cuidados preventivos, já que, ao criarem vínculo com os profissionais, os sujeitos costumam seguir mais suas recomendações, bem como auxilia para obtenção de menores custos ${ }^{18}$.

Quando se pensa em saúde do idoso, é indispensável que se tenha a continuidade do cuidado, ou seja, longitudinalidade da atenção, visto que entre as principais causas de morte nesse grupo estão as doenças do aparelho circulatório, os tumores e as doenças respiratórias ${ }^{19}$. De acordo com a Organização Mundial da Saúde, os maiores eventos da geriatria (depressão, perda cognitiva, incontinência urinária e fratura por quedas) são condições rastreáveis na APS, que devem ser prevenidas, tratadas ou controladas, a fim de evitar morbidades com piores consequências ${ }^{20}$. Dessa forma, é indispensável que se tenha acompanhamento da equipe de saúde ao longo dos ciclos de vida e, também, boas relações entre os profissionais de saúde e os usuários. 
Ao se analisar a orientação à APS dos atributos coordenação e integralidade, observou-se que 44,5\% tiveram orientação em relação à coordenação e apenas $24 \%$ em relação à integralidade. Quando analisados em relação aos diferentes tipos de serviços de saúde, não foram encontradas diferenças significativas. A integralidade implica em unidades de APS que façam arranjos para que o paciente receba todos os tipos de serviços necessários de atenção à saúde, mesmo que esses não sejam feitos dentro da própria unidade, incluindo os encaminhamentos a outros níveis de atenção ou a suportes fundamentais ${ }^{3}$. Já o atributo coordenação refere-se à integração do serviço, ou seja, à forma como será dada a continuidade do paciente, seja pelos profissionais, seja pelo prontuário ou pelo reconhecimento do problema.

Os resultados relacionados à integralidade podem ser justificados pela transição tardia em Porto Alegre das Unidades Básicas de Saúde tradicionais para a Estratégia de Saúde da Família, já que, nessa, o trabalho em equipe funciona dentro dos princípios da saúde da família, o que, consequentemente, pode proporcionar uma atenção diferenciada para a sua população adscrita. Apesar de o Programa Saúde da Família ter sido lançado em 1994, Porto Alegre só aderiu ao programa em 1996, atuando atualmente com 71 unidades de ESF e 101 equipes, que atendem a uma população de 290 mil moradores de um total de 1.416.714 habitantes ${ }^{21}$.

Em relação à coordenação da atenção, é preciso que o sistema funcione por meio das redes de atenção à saúde, pois, para que o atributo coordenação esteja orientado à APS, não depende somente do correto encaminhamento, mas do funcionamento e da integração da rede de atenção. Não só os profissionais, como também os próprios usuários, precisam entender como a rede funciona, para que não trabalhem com foco na agudização das doenças, agindo como um sistema fragmentado. É preciso que se dê continuidade ao cuidado e, também, que haja o reconhecimento de problemas de saúde ${ }^{4}$.

De acordo com Mendes ${ }^{22}$, os sistemas de saúde devem ser pensados de acordo com a saúde da população, sendo o mais conveniente separar as doenças em agudas de curso rápido e crônicas de curso longo, sendo as crônicas responsáveis por $75 \%$ das doenças. O resultado encontrado em relação ao atributo coordenação demonstra as dificuldades que o sistema atual apresenta. Não há prontuários eletrônicos comuns para toda a rede; o sistema de referência e contrarreferência nem sempre é eficaz; existe limitação no número de consultas na APS; os próprios usuários, muitas vezes focados na agudização da doença, acabam procurando diretamente as emergências ou os profissionais especialistas, evitando a porta de entrada ao Sistema de Saúde. Essa realidade dificulta que a APS possa, efetivamente, realizar o seu papel de coordenadora do cuidado.

Embora tenhamos apontado para algumas direções em relação à avaliação da qualidade da APS, estudos longitudinais podem ser úteis para o aprofundamento dessas questões. Da mesma forma, uma pesquisa com amostra composta por idosos moradores de toda a cidade de Porto Alegre, também, poderia proporcionar uma representatividade mais significativa. Além disso, o instrumento PCATool usado neste estudo é voltado para adultos de forma geral e limita-se a algumas perguntas em relação aos problemas de saúde mais prevalentes em idosos, tais como a incontinência urinária e o déficit de memória. Apontamos, igualmente, como limitação possíveis vieses de memória, visto que a amostra estudada foi composta por idosos.

\section{Conclusão}

Este estudo foi pioneiro no que se refere à avaliação da qualidade dos serviços de APS, considerando os diferentes modelos tecnoassistenciais, de acordo com os idosos entrevistados. Os resultados apresentam importantes implicações para as políticas públicas de saúde, visto que as USFs mostraram melhor orientação à APS, com exceção do atributo integralidade, no qual ambas apresentaram a mesma orientação. A maior parte da população residente em Porto Alegre é assistida por Unidades Básicas de Saúde tradicionais. Fica evidente a necessidade de ampliar a cobertura da ESF, de estruturar a rede de atenção bem como de qualificar a atenção à saúde dos idosos que utilizam os serviços de APS na gerência Lomba-Partenon.

\section{Agradecimentos}

À Fundação de Amparo à Pesquisa do Rio Grande do Sul, que financiou o presente estudo por meio do edital 002/2009 PPSUS 2008/2009.

\section{Abstract}

Objective: To assess Primary Health Care (PHC) instruction and to compare the quality of PHC among Basic Health Units (BHUs) and Family Health Units (FHUs) in two districts of Porto Alegre, RS, Brazil. Subjects and method: In this cross-sectional study, we interviewed 402 elderly selected through cluster sampling. Interviews were conducted using a sociodemographic questionnaire in addition to the Primary Care Assessment Tool (PCATool-Brazil). Attributes with scores higher than or equal to 6.6, on a scale from 0 to 10, were found instructed for PHC. The descriptive analysis of variables was performed and the difference of variables between BHUs and FHUs was verified by the chi-square test. Results: From the 402 respondents, $69.7 \%$ were women, average age was 69.18 years, years of education was 4.91 , and income was $R \$ 1,433.84$. The majority (77.9\%) used BHU services and $22.1 \%$ used FHUs. As for the quality of services, $22.9 \%$ were within the recommended by the essential PHC score. When comparing BHUs and FHUs, there was a statistically significant 
difference regarding age $(\leq 70$ year-olds attended FHUs $71.9 \%$ vs. $58.3 \%$ for BHUs) and the following attributes: first contact $(39.7 \%$ BHUs vs. $51.7 \%$ FHUs with PHC instruction, $p=0.044)$ and longitudinality $(68.5 \%$ FHUs $\times 50 \%$ BHUs with PHC instruction, $p=0.02)$. Conclusion: The results show the need for investments on PHC qualification, because even though the Family Health Strategy is prioritized in the reorganization of $\mathrm{PHC}$, it was superior in only two attributes: first contact and longitudinality.

Keywords: Health Care Epidemiology. Health Care for the Elderly. Elderly. Primary Health Care.

\section{Referências}

1. WHO - World Health Organization. Active ageing: a policy framework. Geneva, 2002.

2. Brasil. Ministério da Saúde. Secretaria de Atenção em Saúde. Departamento de Atenção Básica. Manual do instrumento de avaliação da atenção primária à saúde: primary care assessment tool PCATool-Brasil / Ministério da Saúde, Secretaria de Atenção em Saúde, Departamento de Atenção Básica. Brasília: Ministério da Saúde; 2010. 80 p. (Série A. Normas e Manuais Técnicos).

3. Villela WV, Araújo EC, Ribeiro AS, Cuginotti AP, Hayana ET, Brito FC, et al. Desafios da atenção básica em saúde: a experiência da Vila Mariana, São Paulo, Brasil. Cad Saude Publica 2009; 25:1316-24.

4. Starfield B. Atenção Primária: equilíbrio entre necessidades de saúde, serviços e tecnologia. Brasília: UNESCO, MS; 2002.

5. Cassady CE, Starfield B, Hurtado MP, Berk RA, Nanda JP, Friedenberg LA. Measuring consumer experiences with primary care. Pediatrics 2000; 105(4):998-100.

6. Shi L, Starfield B, Jihaong X. Validating of the adult primary care assessment tool. J Family Practice 2001; 50:161-75.

7. IBGE - Instituto Brasileiro de Geografia e Estatística. Sinopse do Censo Demográfico 2010. Rio de Janeiro: IBGE; 2011.

8. Bertolucci PHF, Brucki SMD, Campacci SR, Juliano Y. O mini exame do estado mental em uma população geral: impacto da escolaridade. Arq Neuropsiquiatr 1994; 52:1-7.

9. Harzhein E, Starfield B, Rajmil L, Alvarez-Dardet C, Stein AT. Internal consistency and reliability of Primary Care Assessment Tool (PCATool-Brasil) for child health services. Cad Saude Publica 2006; 22(8):1649-59.

10. Barros FC, Victora CG. Epidemiologia da saúde infantil: um manual para diagnósticos comunitários. 3. ed. São Paulo: Hucitec; Unicef; 1998.

11. Folstein MF, Folstein SE, McHugh PR. "Mini-mental state". A practical method for grading the cognitive state of patients for the clinician. J Psychiatr Res 1975; 12(3):189-98.

12. Chomatas EDRV. Avaliação da presença e extensão dos atributos da Atenção Primária na Rede Básica de Saúde do município de Curitiba, no ano de 2008 [Dissertação de Mestrado]. Porto Alegre: Universidade Federal do Rio Grande do Sul; 2009.

13. Oliveira M. Presença e extensão dos atributos da atenção primária à saúde entre os serviços de Atenção Primária em Porto Alegre: uma análise agregada [Dissertação de Mestrado]. Porto Alegre: Universidade Federal do Rio Grande do Sul; 2007.

14. Brasil. Conselho Nacional de Secretários de Saúde. Atenção Primária e Promoção da Saúde/ Conselho Nacional de Secretários de Saúde. Brasília: Conass; 2007.

15. Gomes KO, Cotta RMM, Cherchiglia ML, Mitre SM, Batista RS. A práxis do agente comunitário de saúde no contexto do programa saúde da família: reflexões estratégicas. Saude Soc 2009; 18(4):744-55.

16. Ditterich RG, Gabardo MCL, Moyses SJ. As ferramentas de trabalho com famílias utilizadas pelas equipes de saúde da família de Curitiba, PR. Saude Soc 2009; 18(3):515-24.

17. Cunha EMD. Vínculo longitudinal na Atenção Primária: avaliando os modelos assistenciais do SUS [Tese de Doutorado]. Rio de Janeiro: Escola Nacional de Saúde Pública; 2009.

18. Hartung P, Tozi A, Silva FJDD, Dantas A, Silva LCOD. Diretrizes de saúde da pessoa idosa. Vitória; 2008. [citado 2012 nov. 8]. Disponível em URL: http://www.saude.es.gov.br/download/34703_DIRETRIZES_PRIMARIAS_SAUDE_MIOLO.pdf.

19. Vasconcelos AMN. Causas de morte em idosos no Brasil. In: XIV Encontro Nacional de Estudos Populacionais: 2004; Caxambu; 2004. 11p.

20. WHO - World Health Organization. Age-friendly Primary Health Care Centres Toolkit. Geneva; 2008.

21. IBGE - Instituto Brasileiro de Geografia e Estatística. Estimativas populacionais dos municípios em 2012. 2012. [citado 2014 nov. 8]. Disponível em URL: http://www.ibge. gov.br/home/presidencia/noticias/noticia_visualiza.php?id_ noticia $=2204 \& i d \_$pagina $=1$.

22. Mendes EV. As redes de atenção à saúde. Cien Saud Colet 2010; 15(5): 2297-305.

\section{Endereço para correspondência:}

Aline Blaya Martins

Rua Ramiro Barcelos, 2.492

90035-003 Porto Alegre, RS

Telefone: (11) 3308 5010/ 93550933

E-mail: alineblaya@hotmail.com

Recebido: 16/09/15. Aceito: 06/06/16. 